\title{
Volatile compounds released by maize grains and silks in response to infection by Fusarium verticillioides and its association with pathogen resistance
}

\author{
C. M. Fauguel ${ }^{a \star}$, V. A. Campos Bermudez ${ }^{b}$ J. Iglesias $^{a}$, M. Fernandez ${ }^{a}$, A. Farroni ${ }^{a}$, \\ C. S. Andreo ${ }^{\text {b }}$ and D. A. Presello ${ }^{\mathrm{a}}$ \\ aInstituto Nacional de Tecnología Agropecuaria, Estación Experimental Agropecuaria Pergamino, C.C. 31. 2700, Pergamino, Pcia, Buenos \\ Aires, and ${ }^{\mathrm{b}}$ Centro de Estudios Fotosintéticos y Bioquímicos (CEFOBI), Suipacha 531, Rosario, Argentina
}

Many plants respond to fungal infections by producing and/or emitting a specific blend of oxylipins, some of them through the lipoxygenase (LOX) pathway. In vitro bioassays revealed that volatiles from silks and grains of a set of six maize genotypes with variable resistance to Fusarium verticillioides affected fungal growth in different ways. Analyses by solid phase microextraction and GC-MS showed different volatile profiles in silks and grains of each genotype. Susceptible genotypes with higher concentrations of precursor polyunsaturated fatty acids produced more volatiles, mainly C9 compounds, in comparison to moderately resistant ones. Real-time PCR from several lipoxygenase transcripts revealed that levels of gene expression depended on the genotype and the inoculation level and suggested that $F$. verticillioides could use compounds from the 9-LOX pathway to promote infection in grains. On the other hand, volatiles produced by the 13-LOX pathway were more important in moderately resistant genotypes and could be associated to field resistance. Results from this set of genotypes indicate that LOX-regulated volatile compounds might be important in F. verticillioides infection and should be targeted in further research to understand disease resistance.

Keywords: Fusarium verticillioides, lipoxygenase, maize, oxylipin, volatile compounds

\section{Introduction}

Fusarium verticillioides (synonym: F. moniliforme; teleomorph: Gibberella moniliformis) causes fusarium ear rot in maize (Zea mays), which is an important disease in Argentina and the world (Chulze et al., 2000; Presello et al., 2007). This pathogen enters into the grain mainly through the silks or grain wounds, affecting yield and grain quality. In addition, the fungus produces mycotoxins, mainly fumonisins, with a large variety of toxicological effects for human and animal health (Marasas, 1995; Hussein \& Brasel, 2001). Developing plant genotypes less susceptible to ear rots is one of the most effective techniques for disease management.

Disease resistance depends on several factors, including biochemical compounds such as complex mixtures of volatile organic compounds (VOCs) released by the plant (e.g. monoterpenes, sesquiterpenes, shikimic acid pathway derivatives such as methyl salicylate, as well as the LOX derivatives, methyl jasmonate and C6-volatiles; Prost et al., 2005; Becker et al., 2014). Some of those VOCs are plant oxylipins, a group of oxygenated lipids derived from polyunsaturated fatty acids (Prost et al.,

*E-mail: fauguel.carolina@inta.gob.ar

Published online 6 February 2017
2005; Christensen \& Kolomiets, 2011). Most oxylipins are produced through the lipoxygenase (LOX) pathway (Feussner \& Wasternack, 2002). Lipoxygenase enzymes catalyse the incorporation of molecular oxygen into free polyunsaturated fatty acids, primarily linoleic (C18:2) and linolenic (C18:3) acids, at either position 9 or 13, giving 9- or 13-hydroperoxides with antimicrobial activities (Howe \& Schilmiller, 2002; Prost et al., 2005; Engelberth et al., 2007; Gao et al., 2008). LOX-derived fatty acid hydroperoxides can be further metabolized into volatile aldehydes and jasmonates in plants by at least seven multienzyme pathways (Mosblech et al., 2009). In maize grains, infections of F. verticillioides induce the synthesis of LOX derivatives promoting or inhibiting fungal development or mycotoxin production (Maschietto et al., 2015).

Oxylipins regulated by 9-LOX and 13-LOX enzymes have antimicrobial activities (Prost et al., 2005; Engelberth et al., 2007; Gao et al., 2008). The pathway catalysed by 13-LOX enzymes leads to the synthesis of alcohols, C6 aldehydes and jasmonates, including jasmonic acid, among others (Feussner \& Wasternack, 2002). The branch involving 9-LOX enzymes is still under study and several works suggest that, contrary to 13-LOX products, these enzymes favour plant infections by pathogens (Gao et al., 2007).

A better knowledge of the importance of VOCs in host-pathogen interactions might accelerate the 
development of hybrids less susceptible to fusarium ear rot. This work aimed to analyse relations between disease resistance and (i) production of VOCs in silks and grain tissues; (ii) effects of VOCs on fungal growth; and (iii) expression of genes related to LOX-9 and LOX-13 VOCs in a group of maize genotypes with variable reaction to F. verticillioides.

\section{Materials and methods}

\section{Plant materials and field assays}

Six maize genotypes, representing a range of variability for disease resistance to fusarium ear rot (Presello et al., 2007), were evaluated after silk inoculation with $F$. verticillioides (Table 1). A complete randomized block design experiment with three replicates was conducted in Pergamino, Buenos Aires Province, Argentina. The six genotypes and two inoculation levels (inoculated with $F$. verticillioides and non-inoculated) were randomized in a $6 \times 2$ factorial arrangement. Each plot consisted of eight $5 \mathrm{~m}$ rows sown at a rate of five plants per metre. P364, a fumonisin-producing isolate of $F$. verticillioides (Iglesias et al., 2010) was used to produce the inoculum. For inoculation, $2 \mathrm{~mL}$ of a conidial suspension $\left(10^{6}\right.$ conidia $\left.\mathrm{mL}^{-1}\right)$ were injected into the silk channel with a cattle vaccinator when silks exhibited brown tips, approximately 4-6 days after silking. Disease severity was visually assessed in each plant as the ear area covered by mould $(\%)$. Data were arcsine-transformed into $\sqrt{[\text { percentage of }}$ disease severity] to normalize errors. Levels of grain fumonisin and ergosterol, a fungal-specific marker molecule to measure fungal biomass, were assessed following Iglesias et al. (2010).

For in vitro experiments, silk and grain samples were collected from 10 plants of each plot. Three days after inoculation, silks were taken from inoculated and non-inoculated plots. A fraction of each sample was used immediately and the remnant fraction was stored at $-80^{\circ} \mathrm{C}$. At maturity $(18-20 \%$ grain moisture), ears were manually harvested from the two central rows of each plot, naturally dried to reach equilibrium moisture (nearly $12 \%$ ), shelled and bulked. Grain samples were stored at $-20{ }^{\circ} \mathrm{C}$ for less than 2 months.

All statistical tests were conducted using INFOSTAT v. 1.1 (Infostat, 2002) at $\alpha=0.05$.

Table 1 Pedigree, origin and Fusarium verticillioides reaction of six Argentinian maize genotypes

\begin{tabular}{|c|c|c|c|}
\hline Genotype & Pedigree & Origin & $\begin{array}{l}\text { Fusarium } \\
\text { reaction }\end{array}$ \\
\hline L4674 & Inbred & $\begin{array}{l}\text { Commercial hybrid } \\
\text { Ax924 }\end{array}$ & Susceptible \\
\hline L4637 & Inbred & LP561 × LP611 & $\begin{array}{l}\text { Moderately } \\
\text { resistant }\end{array}$ \\
\hline $\begin{array}{l}\text { L943 x } \\
\text { L944 }\end{array}$ & $\begin{array}{l}\text { Single cross } \\
\text { between related } \\
\text { inbreds }\end{array}$ & LP915 x LP2541 & $\begin{array}{l}\text { Moderately } \\
\text { resistant }\end{array}$ \\
\hline $\begin{array}{l}\text { L897 x } \\
\text { L900 }\end{array}$ & $\begin{array}{l}\text { Single cross } \\
\text { between related } \\
\text { inbreds }\end{array}$ & LP915 $\times$ LP2541 & Susceptible \\
\hline ARZM05040 & Landrace & $\begin{array}{l}\text { Argentinian germ- } \\
\text { plasm collection }\end{array}$ & $\begin{array}{l}\text { Moderately } \\
\text { resistant }\end{array}$ \\
\hline SPF051 & $\begin{array}{l}\text { Open pollinated } \\
\text { variety }\end{array}$ & $\begin{array}{l}\text { Selection from an } \\
\text { Argentinian germ- } \\
\text { plasm collection }\end{array}$ & $\begin{array}{l}\text { Moderately } \\
\text { resistant }\end{array}$ \\
\hline
\end{tabular}

\section{Grain fatty acid content analysis}

Fatty acid contents in grains were assessed following Borras et al. (2006) with some modifications. The germs of 10 random grains from each genotype were homogenized. The oil from $200 \mathrm{mg}$ flour was extracted in hexane. The method for the preparation of fatty acid methyl esters was performed as described in Official Method Ce 2-66 (AOCS, 1997). A gas chromatograph (Aerograph model 2740-10; Varian Instrument Division) equipped with a flame-ionization detector and a stainless steel Supelco GP 10\% SP-2330 column (cyanosilicone phase) in chromosorb WAW 100/120 mesh $1 / 8$ i.d. (Supelco) was used. The column temperature was set at $200{ }^{\circ} \mathrm{C}$ and the injector and detector ports were set at $220{ }^{\circ} \mathrm{C}$. The flow rate for the $\mathrm{N}_{2}$ carrier gas was $30 \mathrm{~mL} \mathrm{~min}^{-1}$ and the detector gases were set at $30 \mathrm{~mL} \mathrm{~min}^{-1}$ for $\mathrm{H}_{2}$ and $300 \mathrm{~mL} \mathrm{~min}^{-1}$ for air. Peak areas were measured by using a Hewlett Packard model 3365A integrator. The relative amounts of 16:0, 18:0, 18:1 and 18:2 fatty acids were determined as a percentage of the total peak area. The data were mean values of triplicate determination.

\section{In vitro effects of grain and silk volatiles on fungal growth}

Culture media were prepared by adding $15 \mathrm{~mL}$ potato dextrose agar (PDA) to Petri dishes (Zeringue et al., 1996). A $10 \mathrm{~mm}$ agar plug extracted from PDA that had been inoculated with P364 was placed in the well centre. At the same time, milled grain or silk samples $(1 \mathrm{~g})$ were placed in the inverted lid. Inverted Petri dishes were sealed with Parafilm. Control plates were prepared in the same way, but no plant tissue was added. Five replicates of each treatment were assayed. Radial fungal growth was assessed after incubation for 7 days at $25{ }^{\circ} \mathrm{C}$.

\section{Profile of volatiles from silks and grains}

Volatile profiles were assessed in grains from the six genotypes and silks of L4674 and L4637, two inbreds with significant differences in resistance to F. verticillioides. Two grams of milled grains or intact silks were placed into $10 \mathrm{~mL}$ vials. Chlorobenzene (Sigma) was used as the internal standard $\left(50 \mathrm{ng} \mathrm{kg}^{-1}\right.$ final concentration). Vials were sealed with a steel crimp cap fitted with a Teflon/silicone septum conditioned at $100{ }^{\circ} \mathrm{C}$. Vials containing samples were placed in a heating block at $50{ }^{\circ} \mathrm{C}$ with the solid phase microextraction fibre inserted into the headspace above the sample. Adsorption was timed for $50 \mathrm{~min}$. Carboxen/PDMS (Supelco) fibre was used. Fibre was desorbed at $270{ }^{\circ} \mathrm{C}$ for $5 \mathrm{~min}$ on a Varian Saturn 2000 GC-MS system. The initial oven temperature was $40{ }^{\circ} \mathrm{C}$, held for $3 \mathrm{~min}$, ramped at $8{ }^{\circ} \mathrm{C} \min ^{-1}$ to $240{ }^{\circ} \mathrm{C}$ and ramped at $30{ }^{\circ} \mathrm{C} \mathrm{min}^{-1}$ to $290{ }^{\circ} \mathrm{C}$ and held for $5 \mathrm{~min}$. The GC was equipped with a capillary Cpsil $8 \mathrm{~ms}$ column $(0.25 \mathrm{~mm}$ diameter $\times 30 \mathrm{~m}$ length $\times 0.25 \mu \mathrm{m}$ film thickness; Varian) coupled with a capillary VF $17 \mathrm{~ms} \quad(0.25 \mathrm{~mm}$ diameter $\times 30 \mathrm{~m}$ length $\times 0.25 \mu \mathrm{m}$ film thickness; Varian). The injection port was operated in splitless mode with a constant $\mathrm{He}$ carrier gas flow of $1.0 \mathrm{~mL} \mathrm{~min}{ }^{-1}$. Positive identification of component was performed by mass spectral library (NIST, 1998). Integrated areas were normalized on chlorobenzene.

A principal component analysis (PCA) from standardized data was performed using percentages of disease severity, in vitro fungal growth and concentrations of VOCs of each genotype as input traits. 


\section{Effects on fungal growth of silk and grain volatiles}

In vitro experiments were conducted to assess the effects on fungal growth of the most relevant VOCs observed in silk and grain tissues. The set of VOCs included aldehydes (hexanal, trans-2-heptenal, octanal, trans-2-octenal, nonanal), methyl jasmonate, benzothiophene and fatty acid methyl esters (hexanoic acid, heptanoic acid, octanoic acid, decanoic acid, lauric acid, palmitic acid, oleic acid and linoleic acid methyl esters). Fusarium verticillioides was cultured on PDA solid media. Seven millimetre plugs from actively growing regions were taken and placed into the centre of Petri dishes filled with $15 \mathrm{~g}$ PDA. Sterile Eppendorf caps containing 5, 20 or $50 \mu \mathrm{L}$ of the volatiles were placed at the edge of the plate. The PDA dishes were then sealed with Parafilm. After incubation for 7 days at $25^{\circ} \mathrm{C}$, fungal radial growth was assessed. Control plates were prepared as described above without the addition of the volatile compounds. Experiments were conducted in triplicate.

\section{qRT-PCR expression analysis}

Gene expression profiles from LOX genes were analysed by quantitative reverse transcriptase (qRT)-PCR assays in maize silk samples from L4674 and L4637. Gene-specific primers were designed along the last exon and the $3^{\prime}$ UTR region, using PRIMER3 software (Table S1).

Total RNA was extracted from $100 \mathrm{mg}$ of silk pooled samples using the Trizol protocol (Invitrogen) and purified with the RNA Clean-up protocol (QIAGEN) according to the manufacturer's instructions. The amount and the quality of the total RNA were estimated in a NanoDrop spectrophotometer as well as by agarose gel electrophoresis. cDNA was synthesized using $4 \mu \mathrm{g}$ of total RNA and oligo(dT) with Superscript III reverse transcriptase (Invitrogen), according to the manufacturer's instructions, and used for performing qRT-PCR.

Twenty nanograms of single strand cDNA were used for qRT-PCR. SYBR Green PCR Master Mix (Invitrogen) was used for the PCRs according to the manufacturer's protocol, with $2.5 \mathrm{mM} \mathrm{MgCl}_{2}, 0.25 \mu \mathrm{M}$ of each primer and $0.025 \mathrm{U}$ Platinum Taq DNA polymerase (Invitrogen). Relative quantitative analysis was performed using a Stratagene device (Stratagene). Cycling parameters were as follows: initial denaturation at $95^{\circ} \mathrm{C}$ for $2 \mathrm{~min}$; then 45 cycles of $95^{\circ} \mathrm{C}$ for $10 \mathrm{~s}, 58^{\circ} \mathrm{C}$ for $15 \mathrm{~s}$ and $72{ }^{\circ} \mathrm{C}$ for $20 \mathrm{~s}$. Melting curves for each PCR were determined by measuring the decrease of fluorescence with increasing temperature (from 65 to $95^{\circ} \mathrm{C}$ ). The specificity of the PCR was confirmed by melting curve analysis using MxPro Mx3000p software as well as by agarose gel electrophoresis of the products. Relative quantification was normalized using actin1 sequence (MAc1) [EMBL-EBI: J01238] as internal control and the expression ratio was calculated using the $2^{-\Delta \Delta C t}$ method (Livak \& Schmittgen, 2001). Each assay was run in triplicate and repeated at least three times in different samples.

\section{Results}

\section{Disease severity and grain content of ergosterol and} fumonisins

Significant differences in disease severity and grain contents of ergosterol and fumonisins were observed among genotypes (Table 2). Disease severity ranged from 2.2\% to $81.7 \%$ of the ear visibly affected by mould. Grain
Table 2 Ear rot severity, and fumonisin and ergosterol concentrations in grain of six maize genotypes subjected to silk channel inoculation with conidial suspensions of Fusarium verticillioides

\begin{tabular}{lcccc}
\hline & $\begin{array}{l}\text { Disease } \\
\text { severity } \\
(\%)^{\mathrm{a}}\end{array}$ & $\begin{array}{l}\text { Fumonisin } \\
\text { concentration } \\
(\mathrm{ppm})\end{array}$ & $\begin{array}{l}\text { Ergosterol } \\
\text { concentration } \\
(\mathrm{ppm})\end{array}$ & $\begin{array}{l}\text { Disease } \\
\text { reaction }\end{array}$ \\
\hline $\begin{array}{l}\text { Lenotype } \times \\
\text { L944 }\end{array}$ & $4.8 \mathrm{a}$ & $17.7 \mathrm{c}$ & $1.2 \mathrm{a}$ & $\mathrm{MR}$ \\
ARZM05040 & $3.2 \mathrm{a}$ & $19.4 \mathrm{c}$ & $0.9 \mathrm{a}$ & $\mathrm{MR}$ \\
SPF051 & $2.5 \mathrm{a}$ & $4.3 \mathrm{a}$ & $0.6 \mathrm{a}$ & MR \\
L4637 & $2.2 \mathrm{a}$ & $14.4 \mathrm{~b}$ & $4.4 \mathrm{ab}$ & MR \\
L4674 & $81.7 \mathrm{c}$ & $406.6 \mathrm{e}$ & $48.4 \mathrm{c}$ & $\mathrm{S}$ \\
L897 $\times$ & $16.3 \mathrm{~b}$ & $62.9 \mathrm{~d}$ & $7.6 \mathrm{~b}$ & $\mathrm{~S}$ \\
L900 & & & & \\
\hline
\end{tabular}

Means followed by different letters are significantly different at $P<0.05$.

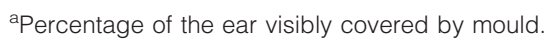

bMR: moderately resistant, S: susceptible.

fumonisin contents ranged from 4.3 to $406.6 \mathrm{ppm}$. Ergosterol concentration, which was used as an indicator of fungal biomass, correlated positively with disease severity $\left(R^{2}=0.98\right)$ and grain fumonisin content $\left(R^{2}=0.99\right)$. These results indicate that, for this isolate of $F$. verticillioides, visual evaluation of disease severity is an accurate parameter to assess fungal invasion and predict mycotoxin accumulation. Genotypes SPF051, ARZM05040, L943 × L944 and L4637 exhibited lower values of disease severity, ergosterol and grain fumonisin contents compared to those of L4674 and L897 × L900 (Table 2), which is consistent with previous information (Presello et al., 2006, 2007, 2008).

\section{Contents of fatty acids in grain}

Grains of susceptible genotypes L897 × L900 and L4674 showed significantly higher amounts of linoleic acid and lower amounts of oleic acid, compared with those of moderately resistant genotypes (Table 3). A significant positive correlation was observed between the grain concentrations of linoleic acid and total volatiles $\left(R^{2}=0.92\right)$, which is consistent with the fact that volatile compounds are synthesized from polyunsaturated fatty acids, such as linoleic or linolenic acid. No associations with disease resistance were observed for the other fatty acids assayed.

\section{In vitro effects of grain and silk volatiles on fungal growth}

Non-inoculated milled grains and silks were used to find differences in fungal growth caused only by constitutive volatiles. Although volatile compounds from infected samples might be important, resistance response from some of these genotypes depends on constitutive mechanisms (Campos-Bermudez et al., 2013), and so the focus was on these. Volatiles generated from milled grains and 
Table 3 Fatty acid composition of grains from six maize genotypes

\begin{tabular}{|c|c|c|c|c|c|c|}
\hline \multirow[b]{2}{*}{ Genotype $^{a}$} & \multicolumn{5}{|l|}{$\%$ Fatty acid ${ }^{b}$} & \multirow[b]{2}{*}{$\begin{array}{l}\text { Linoleic:oleic acid } \\
\text { ratio }\end{array}$} \\
\hline & $\begin{array}{l}\text { Palmitic acid } \\
(16: 0)\end{array}$ & $\begin{array}{l}\text { Estearic acid } \\
(18: 0)\end{array}$ & $\begin{array}{l}\text { Oleic acid } \\
(18: 1)\end{array}$ & $\begin{array}{l}\text { Linoleic acid } \\
(18: 2)\end{array}$ & $\begin{array}{l}\text { Linolenic acid } \\
(18: 3)\end{array}$ & \\
\hline SPF051 (R) & $14.7 \mathrm{c}$ & $1.7 \mathrm{a}$ & $33.8 \mathrm{~cd}$ & 48.7 a & $1.1 \mathrm{~b}$ & $1.44 \mathrm{ab}$ \\
\hline $\begin{array}{l}\text { L943 x L944 } \\
(R)\end{array}$ & $11.7 \mathrm{a}$ & $2.0 \mathrm{ab}$ & $35.1 \mathrm{~d}$ & $50.3 a b$ & $1.0 \mathrm{ab}$ & $1.43 \mathrm{a}$ \\
\hline $\begin{array}{l}\text { ARZM } 05040 \\
(\mathrm{R})\end{array}$ & $13.6 \mathrm{~b}$ & $2.1 \mathrm{~b}$ & $32.3 \mathrm{bc}$ & $51.1 \mathrm{~b}$ & $0.9 a b$ & $1.58 b$ \\
\hline L4637 (R) & $13.2 \mathrm{~b}$ & $2.0 \mathrm{ab}$ & $32.7 \mathrm{bc}$ & $51.4 \mathrm{~b}$ & $0.8 \mathrm{a}$ & $1.57 \mathrm{~b}$ \\
\hline $\begin{array}{l}\mathrm{L} 897 \times \mathrm{L} 900 \\
(\mathrm{~S})\end{array}$ & $11.3 \mathrm{a}$ & $1.7 \mathrm{a}$ & $31.0 \mathrm{~b}$ & $55.3 \mathrm{c}$ & $1.0 \mathrm{ab}$ & $1.78 \mathrm{c}$ \\
\hline L4674 (S) & $11.2 \mathrm{a}$ & $1.8 \mathrm{a}$ & $27.3 \mathrm{a}$ & $58.6 \mathrm{~d}$ & $1.2 \mathrm{~b}$ & $2.15 \mathrm{~d}$ \\
\hline
\end{tabular}

${ }^{a} \mathrm{R}$, moderately resistant; $\mathrm{S}$, susceptible to field Fusarium verticillioides infection.

${ }^{b}$ Means followed by different letters are significantly different at $P<0.05$ ( $t$-test).

silks of each genotype exhibited differences in biological activities after exposure to solid cultures of F. verticillioides over 7 days. Fungal growth in Petri dishes was reduced by volatiles released from grains or silks of SPF051 and L4637 moderately resistant genotypes, but promoted by those released from silk or grains of L4674 and L897 × 900 susceptible genotypes (Table 4; Fig. 1). In vitro inhibition and enhancing effects for these genotypes were observed regardless of the tissue used for the assays. No effects were found for volatiles released from silks and grains of ARZM05040 and L943 × L944.

\section{Volatile compounds from grains and silks}

In order to identify VOCs that may be responsible for the bioactivity observed in vitro, GC-MS chromatographic profiles were analysed in grain samples from inoculated and non-inoculated treatments of the six maize genotypes and silk samples of L4674 and L4637.

\section{Volatile compounds in grains}

Seventy-two VOCs (Table S2) were identified, which consisted of alcohols, aldehydes, ketones, alkenes, carboxylic acids, sulphur-containing compounds and terpenoids,

Table 4 Effects on radial growth of 7-day-old cultures of Fusarium verticillioides exposed to volatiles released by silk or grain tissues from six maize genotypes

\begin{tabular}{lll}
\hline & \multicolumn{2}{l}{ Fungal radial growth $(\mathrm{cm})^{\mathrm{a}}$} \\
\cline { 2 - 3 } Genotype & Milled kernel & Silks \\
\hline SPF051 & $1.97 \mathrm{a}$ & $2.06 \mathrm{a}$ \\
L4637 & $2.02 \mathrm{a}$ & $2.11 \mathrm{a}$ \\
L943 $\times$ L944 & $2.51 \mathrm{bc}$ & $2.19 \mathrm{ab}$ \\
ARZM05040 & $2.61 \mathrm{bcd}$ & $2.41 \mathrm{~cd}$ \\
L4674 & $2.72 \mathrm{~cd}$ & $2.55 \mathrm{de}$ \\
L897 $\times$ L900 & $2.81 \mathrm{~d}$ & $2.67 \mathrm{e}$ \\
Control & $2.36 \mathrm{~b}$ & $2.27 \mathrm{bc}$ \\
\hline
\end{tabular}

aMeans followed by different letters are significantly different at $\alpha=0.05$. among others. Chromatographic patterns showed quantitative and qualitative differences among genotypes and inoculation levels. A PCA analysis based on disease severity, fungal growth and volatile concentrations revealed three major areas of the graph, and its distribution did not appear to be independent of genotype and inoculation level (Fig. 2). The relative contents of the most significant volatiles released by grains of the six genotypes in inoculated and non-inoculated treatments are displayed in Figure 3. From the PCA analysis it was observed that Group 1 included a large number of compounds released by L4674, regardless of the inoculation level, and by L897 $\times$ L900 after inoculation. The large size of the vectors in PC1, and the position of L4674 at the extreme of the axes, indicate that this genotype produced large amounts of this set of compounds. Vectors for disease severity and fungal growth were close to this group, indicating that these compounds might be associated with fungal growth promotion. The compounds grouped in this sector included several C9-compounds such as nonanal (V16), (E,E)-2,6nonadienal (V24), 1-nonene (V32), (E)-2-nonen-1-ol (V34), 6-nonenal (V39) and 8-oxo-nonenal (V66). Group 2 was mainly defined by scores in PC2 and included volatiles released by moderately resistant genotypes SPF051 and L4637 without inoculation. This sector mostly included aromatic compounds such as benzothiophene (V47), naphthalene (V51), 2-methyl naphthalene (V53), 2,2-dimethyl-propanoic acid (V18) and benzoic acid (V42). Upon inoculation, these genotypes tended to generate a new class of volatile compounds (Group 3) including methyl jasmonate (V72). They were grouped at the other extreme of the PC1 axis and corresponded with several compounds released by the resistant genotypes and noninoculated L897 $\times$ L900. According to their position in Figure 2, these compounds seem to be present in low concentrations in grains of L4674, the most susceptible line.

\section{Volatile compounds in silks}

Moderately resistant L4637 and susceptible L4674 genotypes that had exhibited differences in disease resistance and volatile effects on in vitro fungal growth were 

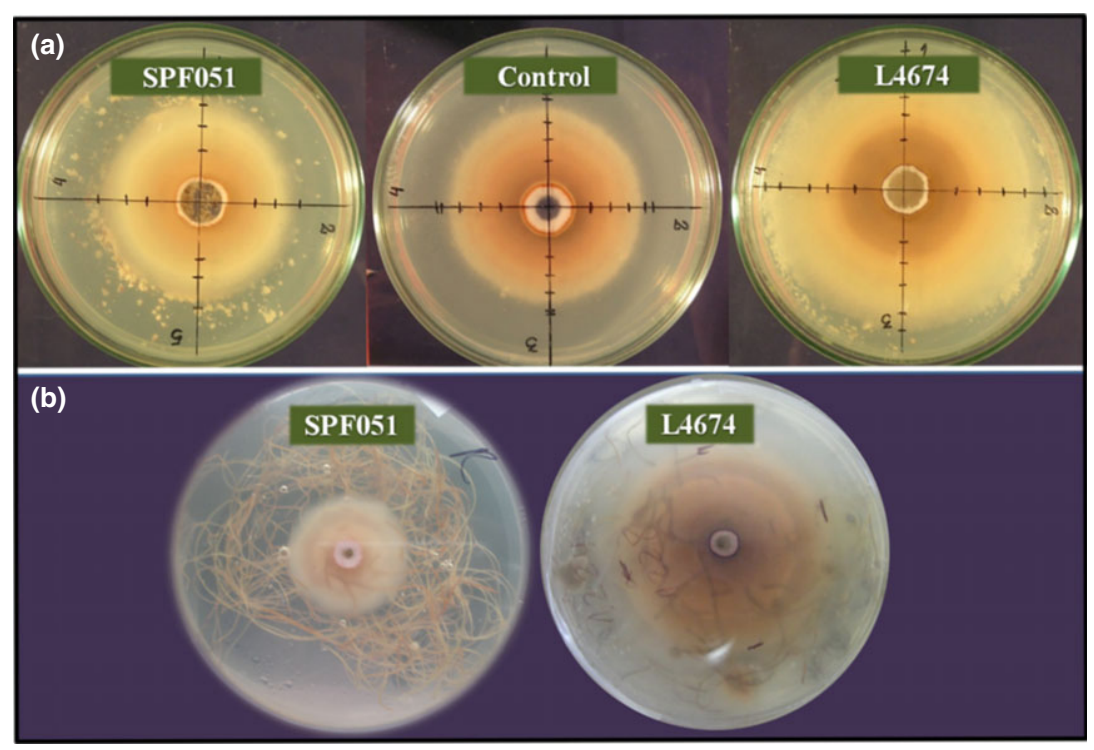

Figure 1 Fusarium verticillioides growth in Petri plate exposed to volatiles released from $1 \mathrm{~g}$ of milled grains (a) and silks (b) from field resistant (SPF051) and field susceptible (L4674) maize genotypes. The photograph was taken 7 days after inoculation with a conidial suspension of $F$. verticillioides into the centre well of the Petri plate. [Colour figure can be viewed at wileyonlinelibrary. com].

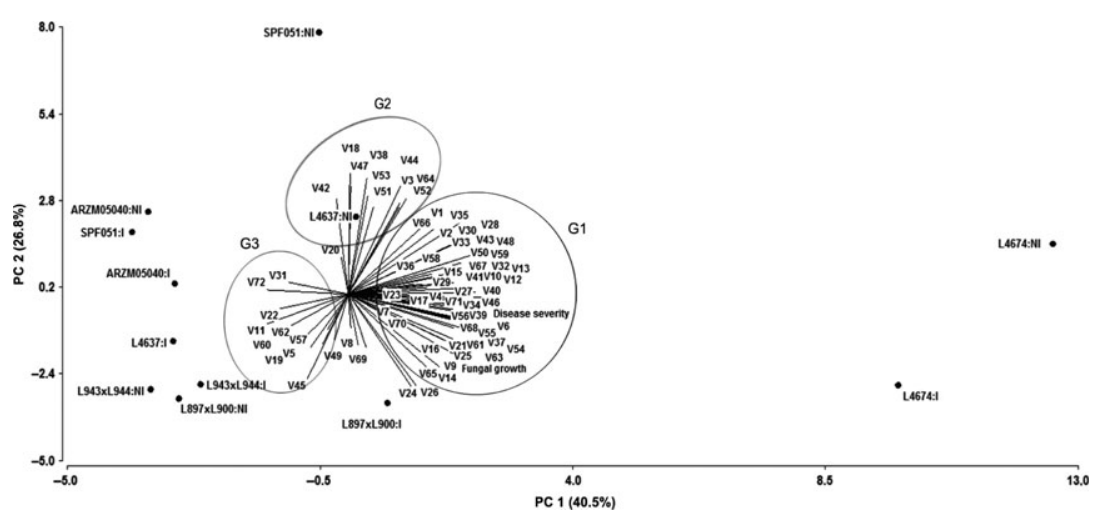

Figure 2 Principal component analysis from the volatile compounds released by grains of six maize genotypes inoculated (I) with Fusarium verticillioides and non-inoculated (NI). G1: Group 1, G2: Group 2, G3: Group 3. evaluated. The chromatographic pattern analysis of VOCs obtained by GC-MS showed quantitative and qualitative differences between genotypes. Forty-three VOCs were identified, including alcohols, aldehydes, carboxylic acid, benzoic acids, terpenoids and fatty acid derivatives, among others (Table S3).

In the PCA analysis, the two first principal components explained $80.2 \%$ of the total variance (Fig. 4). Inoculation levels were discriminated by scores in PC1 and genotypes by scores in PC2. Volatile compounds with higher scores in PC1 represented most of the variation, indicating that F. verticillioides inoculation caused a stimulation of volatile liberation. The most important volatiles from this group were oxazole [2-(1-naphthalenyl)-5-phenyl] (V17), isojasmone (V23), 2-hexyl-1decanol (V4), nonanoic acid, 9-oxo-, methyl ester (V22), heneicosene (V3), 1-eicosanol (V2), nonadecane (V5), oleic acid, methyl ester (V10), hexadecanoic acid, 14methyl-, methyl ester (V13), isocaryophyllene (V26), linoleic acid, methyl ester (V12) and nonadecanoic acid, 18-oxo-, methyl ester (V18). The relative content of the most significant volatiles released by silks of the two genotypes in inoculated and non-inoculated treatments are shown in Figure 5. Some compounds, including 2,4,4-trimethyl-1-hexene (V37), 7-methyl-5-octen-4-one (V36), naphthalene (V39) and hexanal (V41), exhibited lower scores in PC1 (i.e. exhibited prevalence in the noninoculated treatment). Hexanal was released by the noninoculated resistant genotype (Fig. 4). Scores in PC2 indicated that inoculated susceptible and resistant genotypes produced different classes of volatiles. L4637 showed higher concentrations of C6 volatiles, including trans-2octenal (V8) and trans-2-hexenal (V43), volatiles derived from the 13-LOX pathway and (E,E)-2,4-nonadienal (V35) and (Z)-6-nonenal (V7), which are derived from the 9-LOX pathway. These results indicate a stimulation of both 9- and 13-LOX pathways. This moderately resistant line also released fatty acid methyl and ethyl esters, such as linoleic acid, methyl ester (V6), linoleic acid, ethyl ester (V12) and oleic acid, methyl ester (V10); 4methyl-2-propyl-furan (V34); 1,3,5-cycloheptatriene (V42) and 2,2,4-trimetyl-3-penten-1-ol (V28). On the other hand, inoculated silks of L4674 released alkylderived fatty acids, such as methyl ester fatty acids like 


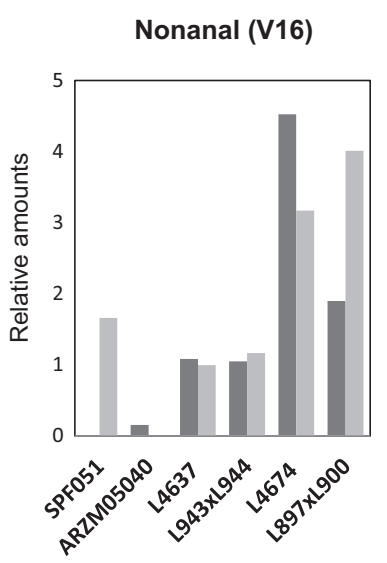

6-nonenal (V39)

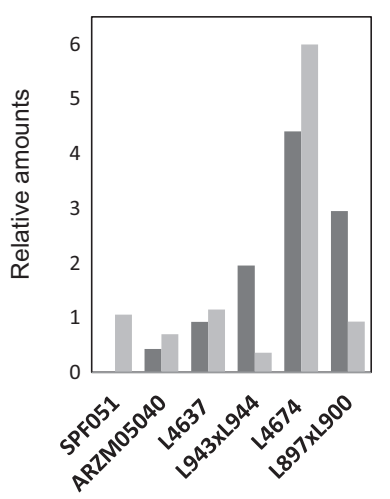

2,2-dimethyl-propanoic acid

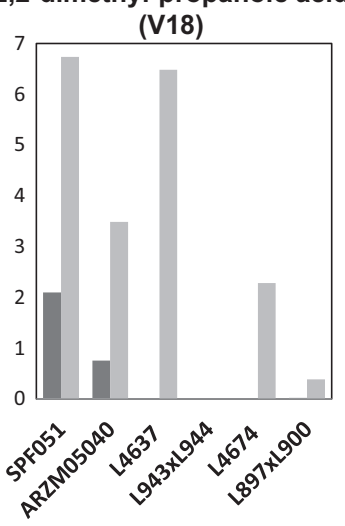

Benzothiophene (V47)

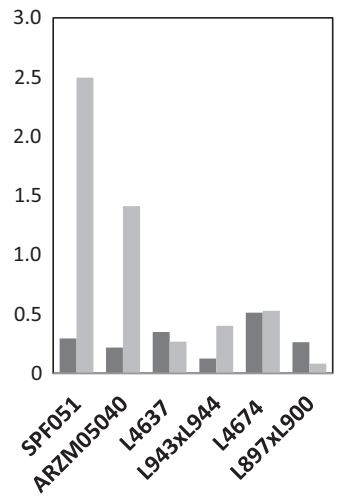

(E,E)-2,6-nonadienal (V24)

(E)-2-nonen-1-ol (V34)
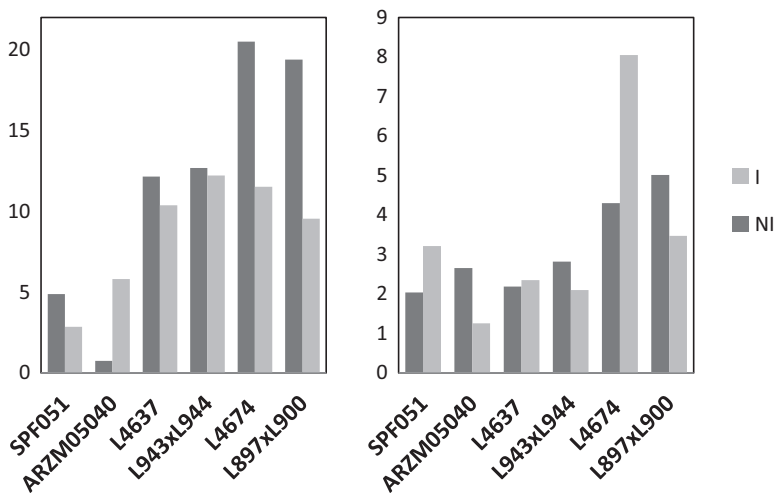

2-methyl naphthalene (V53) Methyl jasmonate (V72)

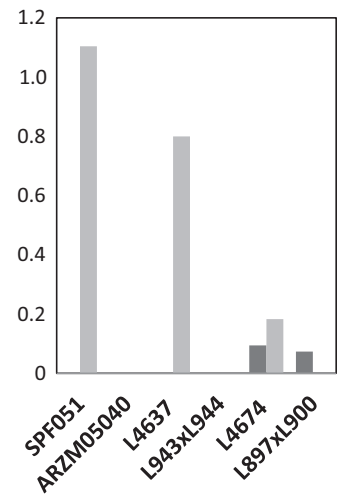

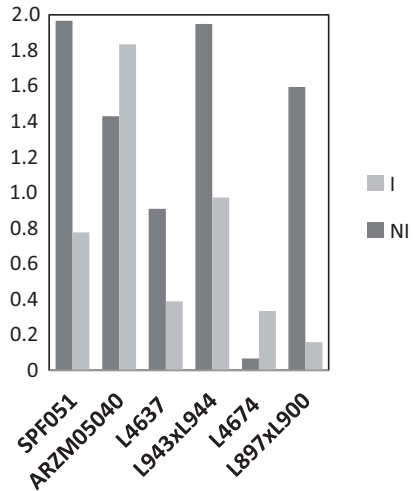

Figure 3 Relative content of the most significant volatiles released by grains of six maize genotypes inoculated (I) with Fusarium verticillioides and non-inoculated (NI).

6,11-eicosadienoic acid, methyl ester (V11), hexadecanoic acid, 14-methyl-, methyl ester (V13), 9-dodecenoic acid, methyl ester (V14), nonadecanoic acid-, 18oxo, methyl ester (V18), nonanoic acid, 9-oxo-, methyl ester (V22), 2-methyl decanoic acid (V31), germacrene B (V25) and aromatic hydrocarbons like naphthalene (V39) and azulene (V38).

In vitro effects of volatile compounds on fungal growth

In vitro experiments were conducted to test effects on fungal growth of the VOCs possibly involved in disease reaction. Fungal growth was affected not only by the type of volatile but also by the volatile concentration (Fig. 6). Aldehydes exhibited more inhibition effect than methyl esters. A complete inhibition of F. verticillioides growth was observed after addition of several amounts of trans-2-octenal. Trans-2-heptenal and nonanal showed a marked inhibitory effect on fungal growth with higher efficiency at low concentrations. The addition of $50 \mu \mathrm{L}$ of hexanal, and 20 and $50 \mu \mathrm{L}$ of octanal also reduced fungal growth. From the analysis of volatiles that, according to PCA, seemed to be associated with disease resistance, it was found that benzothiophene inhibited fungal growth, but no effect was observed for methyl jasmonate regardless of the amount added. From the methyl esters assayed, only oleic acid showed no effect on fungal growth. The other methyl esters (hexanoic acid, heptanoic acid, octanoic acid, decanoic acid, lauric acid and palmitic acid methyl ester) displayed low inhibition effects.

\section{Silk lipoxygenase expression in response to pathogen infection}

Earlier reports have indicated that oxylipins produced by the LOX pathway are involved in plant-pathogen interactions (Burow et al., 2000; Gao et al., 2007; Christensen \& Kolomiets, 2011; Maschietto et al., 2015). Here, LOX gene expression from maize silks was analysed to compare gene expression with volatile profiles from inoculated and non-inoculated treatments of L4637 and L4674.

Several transcripts described as lipoxygenase were identified from the Maize Genetics and Genomics Database (http://www.maizegdb.org). Among them, six genes were chosen based on previous studies $(\mathrm{ZmLOX} 4,5,6$, 7, 10 and 11). ZmLOX4 and 5 are predicted to be type 9-LOXs (Park et al., 2010; Maschietto et al., 2015) whereas ZmLOX7, 10 and 11 (Nemchenko et al., 2006) 


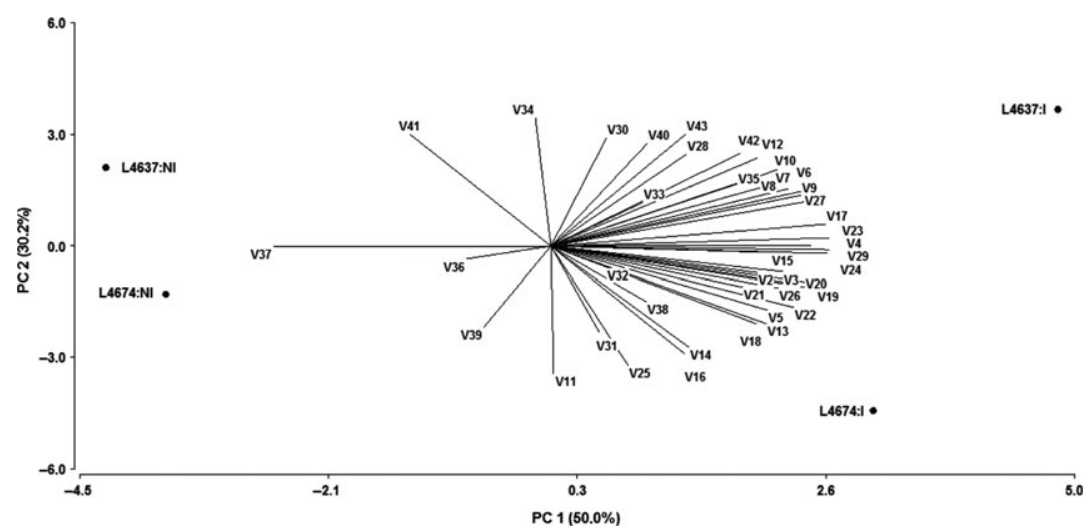

Figure 4 Principal component analysis from the volatile compounds released by silks of two maize inbreds L4637 and L4674 inoculated (I) with Fusarium verticillioides and non-inoculated (NI). Vectors stand for the expression of each volatile.
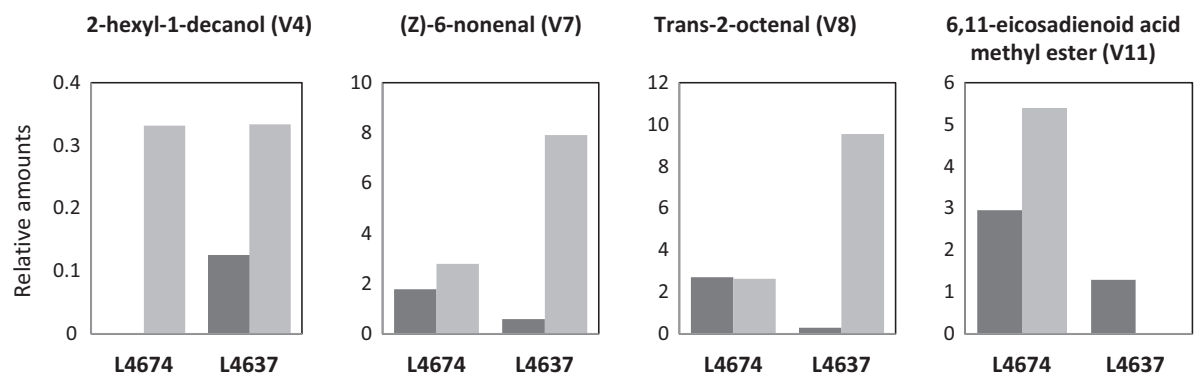

Linoleic acid ethyl ester (V12)
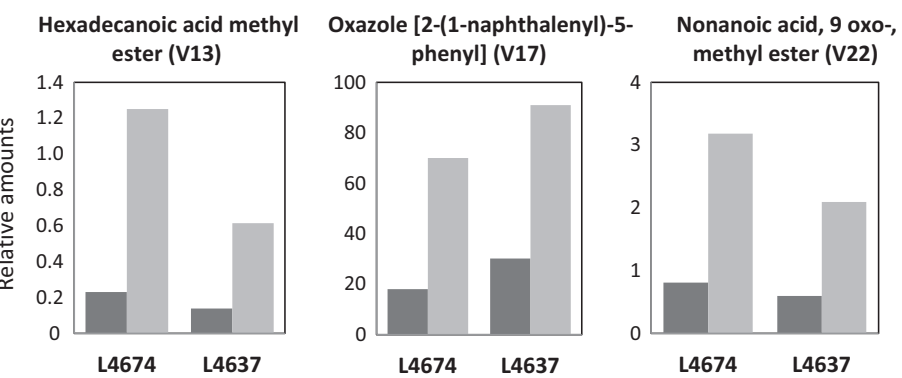

Isojasmone (V23)

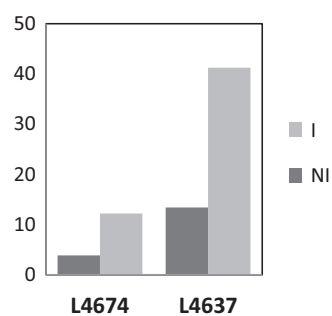

4,4-trimethyl-1-hexene
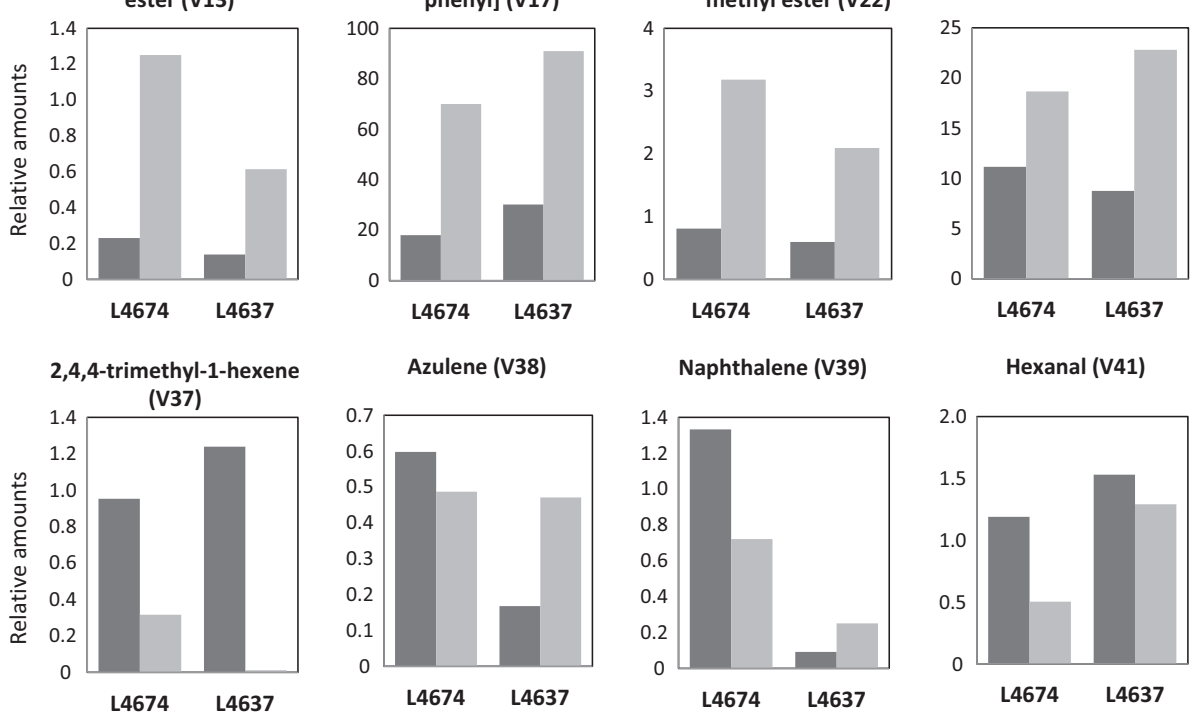

(E,E) 2,4 nonadienal (V35)
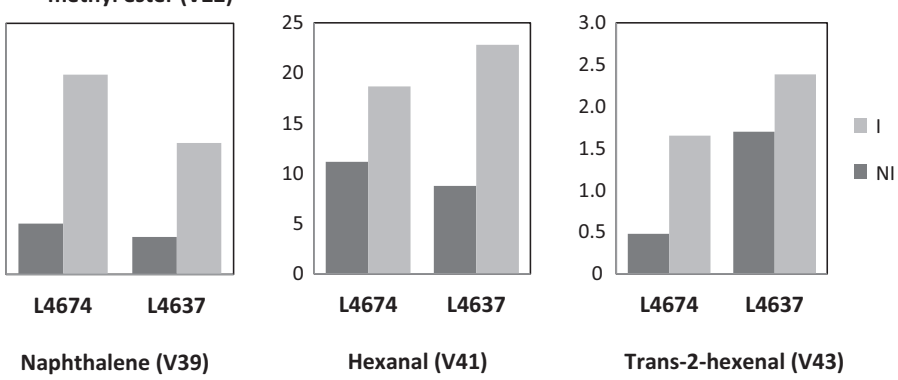

Hexanal (V41)

Trans-2-hexenal (V43)
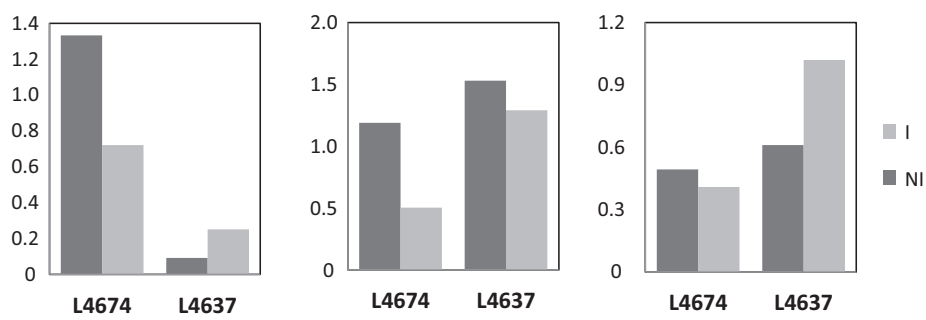

Figure 5 Relative content of the most significant volatiles released from silks of two maize genotypes inoculated (I) with Fusarium verticillioides and non-inoculated (NI).

are predicted to be 13-LOXs. Conversely, ZmLOX6 has been reported to be a hydroperoxide lyase enzyme and seems to be involved in a branch of the 13-LOX pathway (Gao et al., 2008).

From the qRT-PCR analysis, it was observed that 9LOXs genes, ZmLOX4 and ZmLOX5, were induced after fungal inoculation in both genotypes, with higher expression in the L4674 (Fig. 7). On the other hand, ZmLOX6 transcript was induced after inoculation in both lines, with higher expression in L4637. ZmLOX7 expression was induced by inoculation in L4674 only, but higher basal expression levels were observed in 

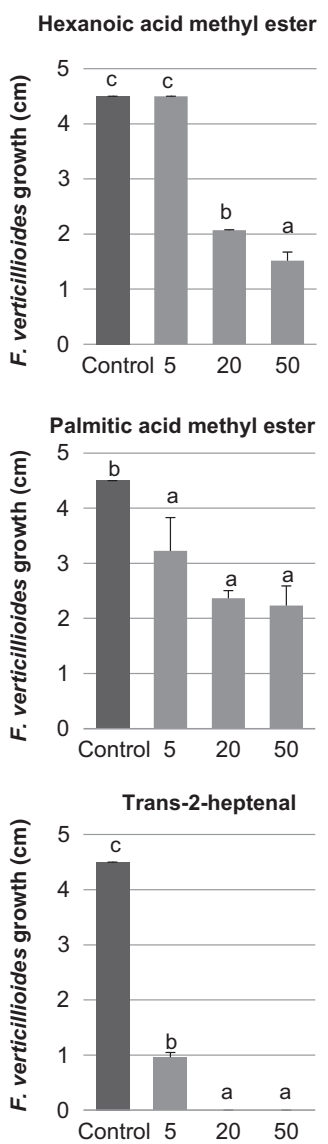
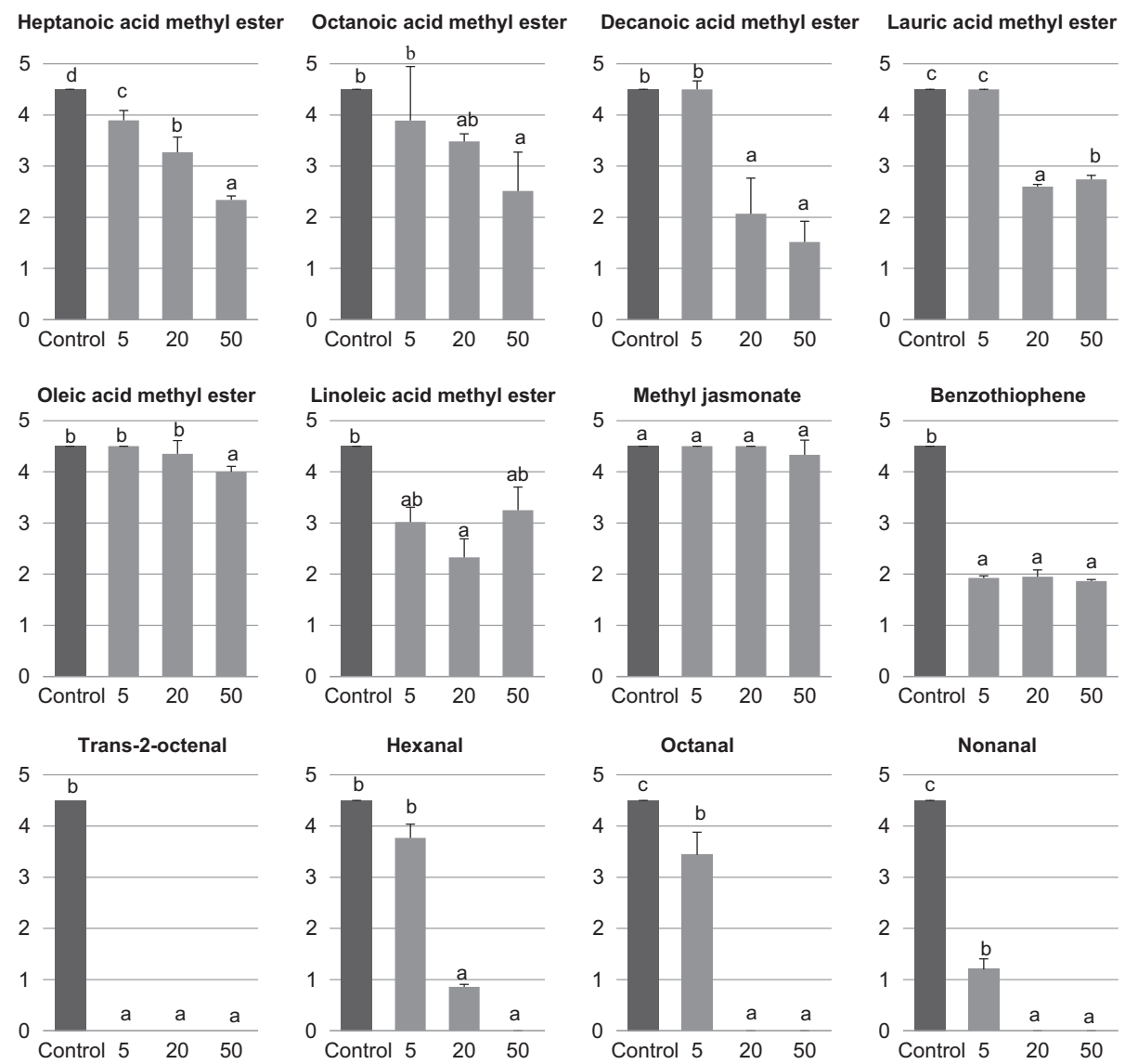

Figure 6 In vitro growth of Fusarium verticillioides after 7 days of continuous exposure to variable amounts (5, 20 and $50 \mu \mathrm{L})$ of volatile compounds. Columns with different letters indicate significant differences between means at the level of $P<0.05$.

L4637. Expression of ZmLOX10 and ZmLOX11 genes was affected by inoculation. While both genes were induced by inoculation in L4637 only, expression of $\mathrm{ZmLOX} 10$ seemed to be repressed after inoculation of L4674. These results indicate that 13-LOXs gene expression seems to be higher in the moderately resistant inbred while 9-LOXs gene expression, which was stimulated by inoculation, is higher in the susceptible inbred.

\section{Discussion}

Plants are capable of defending themselves by releasing complex mixtures of VOCs. Plant oxylipins, produced via the LOX pathway, are some VOCs released by plants as signals in defence and development (Prost et al., 2005; Piesik et al., 2015). In vitro experiments, where fungal growth was assessed after exposure to volatiles from silks or grains, showed that VOC blends released by each genotype affected fungal growth in different ways. Volatile blends from silks and grains of the most resistant genotypes inhibited, and those of the susceptible genotypes promoted, fungal growth. Those effects were observed regardless of the tissue, indicating that biological activity of volatiles is important in silks and grains.
Chromatographic analysis performed in grains of all genotypes and silks of L4637 and L4674 revealed differences in amount and profiles of volatiles released by each genotype. Principal component analyses using grain volatile profiles, disease severity and in vitro fungal growth as input traits showed that volatile profile affected fungal growth. Considering that vectors for disease severity and fungal growth from PCA were closely related, and that the effects of volatile blends on fungal growth were similar in silks and grains, which can be colonized by Fusarium, volatile production might be a factor affecting field disease resistance.

The most susceptible genotypes, such as L4674, produced large amounts of VOCs with prevalence of C9 compounds, indicating that these volatiles might be associated with fungal growth promotion observed in Petri dishes. Thus, fungal growth could be positively regulated by 9 -LOX products affecting susceptibility of the maize plant to F. verticillioides. These results are consistent with previous research on aspergillus ear rot reporting that 9-LOX-derived compounds induce aflatoxin biosynthesis in Aspergillus species (Burow et al., 2000). For fusarium ear rot, evidence from a study of a 9-LOX gene mutant in maize, $\mathrm{ZmLOX3}$, also supported this hypothesis, showing that the deficiency in 9-LOX derivatives 


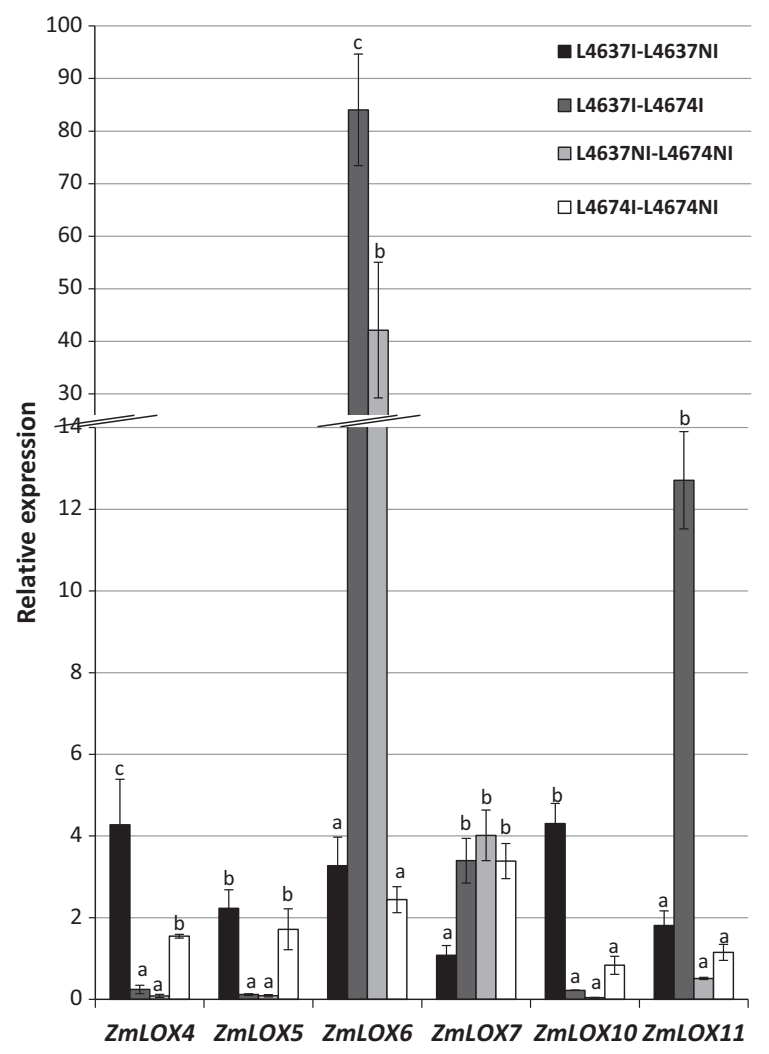

Figure 7 Relative expression of the transcripts of $Z m L O X$ genes in silks of moderately resistant $L 4637$ and susceptible $L 4674$ maize genotypes. NI: non-inoculated, I: inoculated with Fusarium verticillioides. Columns with different letters indicate significant differences between means at the level of $P<0.05$.

significantly reduces production of pathogenic spores and mycotoxins of F. verticillioides in maize seeds (Gao et al., 2007).

In studies of grain volatiles, moderately resistant L4637 and SPF051 were grouped by PCA in a cluster of genotypes producing grain aromatic VOCs, such as benzothiophene, naphthalene, 2-methyl naphthalene and benzoic acid. These compounds and their derivatives have antifungal effects in different species (Pinto et al., 2008; Kim et al., 2010; Salae et al., 2010). Inoculation induced the synthesis of methyl jasmonate, a phytohormone inducing the expression of defence genes in plants in response to attack by several pathogens (Penninck et al., 1998; Turner et al., 2002) in these genotypes. In silk volatile experiments, most volatiles were produced after inoculation, indicating that the fungus caused a stimulation of volatile production. Inoculation increased the production of hexanal and trans-2-hexenal volatiles, particularly in silks from L4637, suggesting the contribution of 13-LOX pathway-derived volatile aldehydes in infection response. For aspergillus ear rot, downstream derivatives of 13-LOX, including methyl jasmonate and various C6-C12 alkanal and alkenal volatiles (Zeringue et al., 1996; Mosblech et al., 2009) also seem to be involved in inhibiting aflatoxin production.
The results here indicate that the 9-LOX pathway is activated in grains of susceptible genotypes, suggesting an important role of this pathway in susceptibility to F. verticillioides. Based on real-time PCR results, it seems that 9-LOX genes $(\mathrm{ZmLOX} 4$ and $\mathrm{ZmLOX5})$ were triggered in response to pathogen attack in both genotypes, with higher expression levels in the susceptible line. Park (2011) reported that $Z m L O X 5$ contributes to susceptibility of maize to Colletotrichum graminicola, a leaf fungal pathogen. In contrast, it was found that both lox 4 and lox5 mutants enhanced susceptibility to grain colonization and conidia production of F. verticillioides. These results are consistent with those from PCA indicating that the growth of $F$. verticillioides and fumonisin biosynthesis could be positively regulated by 9-LOXs and may be involved in susceptibility of the maize plant to this fungus. On the other hand, ZmLOX10 transcript was induced after inoculation in L4637, indicating a possible role for LOX10 in disease defence. Furthermore, ZmLOX6 transcript showed higher expression in L4637, indicating that this gene could also contribute to a resistance response. Conversely, Gao et al. (2008) reported that this gene was strongly induced by the fungal pathogen Cochliobolus carbonum during compatible interactions, suggesting that $\mathrm{ZmLOX6}$ may contribute to pathogen invasion. Therefore, further investigations are needed in order to determine the possible defence role of 13-LOX pathway derivatives in plants.

In vitro evaluations of the effect of specific volatiles on fungal growth revealed that aldehydes exhibited more inhibition effect than methyl esters. A complete inhibition of fungal growth was observed upon the addition of trans2-octenal. Trans-2-heptenal and nonanal aldehydes showed high efficiency at low concentrations. It was found that benzothiophene inhibited fungal growth and no effect was observed for methyl jasmonate. This is consistent with previous results indicating antifungal effects of benzothiophene (Pauletti et al., 2000). The absence of fungal growth inhibition for methyl jasmonate may be due to the fact that this compound is involved in signalling of defence pathways (Penninck et al., 1998; Turner et al., 2002) more than in specific inhibition mechanisms. These in vitro experiments show that several compounds have a direct inhibitory effect on fungal growth and could be targeted for further studies on their activities to prevent fungal infections in natural conditions.

This study indicates a correlation between VOC production and the grain profiles of fatty acids, which are volatile precursors. The most susceptible genotypes exhibited higher contents of linoleic acid and higher production of VOCs compared to those of moderately resistant genotypes. Recent studies have shown that the composition of total fatty acids can influence infections of $F$. verticillioides and fumonisin accumulation in maize under field conditions (Scala et al., 2014). Similar results were reported by Dall'Asta et al. (2012) who found that hybrids with higher linoleic acid content also showed high fumonisin contamination. Thus, for the set of maize genotypes in the present study, concentration 
of polyunsaturated fatty acids seems to affect disease resistance.

The results from these experiments indicate that the amounts and profiles of volatiles produced in silks and grains depends on the genotype and infection level, and seem to play a role in differences in disease resistance observed in this set of maize genotypes. The most resistant genotypes produce blends of VOCs that might be responsible for low disease severities observed in field conditions, and the susceptible genotypes produce VOCs promoting fungal growth, mostly via the 9-LOX pathway. This is new evidence for the role of specific 9-LOXs and their derivatives as possible factors affecting ear rot severity in maize. This result provides evidence that fungal infection changes plant LOX gene expression, leading to possible alterations in the fungal-host interaction.

\section{Acknowledgements}

This work was supported by grants from the Instituto Nacional de Tecnología Agropecuaria (INTA PNCYO1127023 and PNCYO-1127043), the Agencia Nacional de Promoción Científica y Tecnológica (ANPCyT) PICT 358 and the Secretaria de Estado de Ciencia, Tecnología e Innovación de Santa Fe (Sectei). The authors thank the maize breeding and the plant pathology teams at INTA for assistance with field experiments. They also are grateful to Mabel Percibaldi and Román Dilello for the chromatographic fatty acid content analysis. The authors have no conflict of interest to declare.

\section{References}

AOCS, 1997. Official Methods and Recommended Practices of the American Oil Chemists' Society: Method Ce 2-66. GLC Ranges of Fatty Acid Composition. Champaign, IL, USA: AOCS Press.

Becker E, Herrfurth C, Irmisch S et al., 2014. Infection of corn ears by Fusarium spp. induces the emission of volatile sesquiterpenes. Journal of Agricultural and Food Chemistry 62, 5226-36.

Borras F, Seetharaman K, Yao N, Robutti JL, Percibaldi NM, Eyhérabide GH, 2006. Relationship between popcorn composition and expansion volume and discrimination of corn types by using zein properties. Cereal Chemistry 83, 86-92.

Burow GB, Gardner HW, Keller NP, 2000. A peanut seed lipoxygenase responsive to Aspergillus colonization. Plant Molecular Biology 42, 689-701.

Campos-Bermudez VA, Fauguel CM, Tronconi MA, Presello DA, Andreo CS, 2013. Transcriptional and metabolic changes associated to the infection by Fusarium verticillioides in maize inbreds with contrasting ear rot resistance. PLOS ONE 8, e61580.

Christensen SA, Kolomiets MV, 2011. The lipid language of plant-fungal interactions. Fungal Genetics and Biology 48, 4-14.

Chulze SN, Ramirez ML, Torres A, Leslie JF, 2000. Genetic variation in Fusarium section Liseola from no-till maize in Argentina. Applied and Environmental Microbiology 66, 5312-5.

Dall'Asta C, Falavigna C, Galaverna G, Battilani P, 2012. Role of maize hybrids and their chemical composition in Fusarium infection, fumonisin production and masking. Journal of Agricultural and Food Chemistry 60, 3800-8.

Engelberth J, Seidl-Adams I, Schultz JC, Tumlinson JH, 2007. Insect elicitors and exposure to green leafy volatiles differentially upregulate major octadecanoids and transcripts of 12-oxo phytodienoic acid reductases in Zea mays. Molecular Plant-Microbe Interactions 20, 707-16.

Feussner I, Wasternack C, 2002. The lipoxygenase pathway. Annual Review of Plant Biology 53, 275-97.

Gao X, Shim WB, Göbel C et al., 2007. Disruption of a maize 9lipoxygenase results in increased resistance to fungal pathogens and reduced levels of contamination with mycotoxin fumonisin. Molecular Plant-Microbe Interactions 20, 922-33.

Gao X, Starr J, Göbel C et al., 2008. Maize 9-lipoxygenase ZmLOX3 controls development, root-specific expression of defense genes, and resistance to root-knot nematodes. Molecular Plant-Microbe Interactions 21, 98-109.

Howe GA, Schilmiller AL, 2002. Oxylipin metabolism in response to stress. Current Opinion in Plant Biology 5, 230-6.

Hussein HS, Brasel JM, 2001. Toxicity, metabolism, and impact of mycotoxins on humans and animals. Toxicology 167, 101-34.

Iglesias J, Presello DA, Botta G, Lori GA, Fauguel CM, 2010. Aggressiveness of Fusarium Section Liseola isolates causing maize ear rot in Argentina. Journal of Plant Pathology 92, 205-11.

Infostat, 2002. infostat Version 1.1. Grupo Infostat. F.C.A. Córdoba, Argentina: Universidad Nacional de Córdoba.

Kim JH, Campbell BC, Mahoney N, Chan KL, Molyneux RJ, Balajee A, 2010. Augmenting the activity of antifungal agents against aspergilli using structural analogues of benzoic acid as chemosensitizing agents. Fungal Biology 114, 817-24.

Livak KJ, Schmittgen TD, 2001. Analysis of relative gene expression data using real-time quantitative PCR and the $2^{-\Delta \Delta C t}$ method. Methods 25, 402-8.

Marasas WFO, 1995. Fumonisins: their implications for human and animal health. Natural Toxins 3, 193-8.

Maschietto V, Marocco A, Malachova A, Lanubile A, 2015. Resistance to Fusarium verticillioides and fumonisin accumulation in maize inbred lines involves an earlier and enhanced expression of lipoxygenase (LOX) genes. Journal of Plant Physiology 188, 9-18.

Mosblech A, Feussner I, Heilmann I, 2009. Oxylipins: structurally diverse metabolites from fatty acid oxidation. Plant Physiology and Biochemistry 47, 511-7.

Nemchenko A, Kunze S, Feussner I, Kolomiets M, 2006. Duplicate maize 13-lipoxygenase genes are differentially regulated by circadian rhythm, cold stress, wounding, pathogen infection, and hormonal treatments. Journal of Experimental Botany 57, 3767-79.

NIST, 1998. NIST 98 Mass spectral library with Windows search program (version 1.7). NIST Standard Reference Database 1A. [chemdata.nist.gov/mass-spc/ftp/mass-spc/v1_7/Ver17Man.doc]. Accessed 8 December 2016.

Park YS, 2011. Diverse Functions of the Two Segmentally Duplicated 9Lipoxygenases ZmLOX4 and ZmLOX5 of Maize. College Station, TX, USA: Texas A\&M University, PhD thesis.

Park YS, Kunze S, Ni X, Feussner I, Kolomiets MV, 2010. Comparative molecular and biochemical characterization of segmentally duplicated 9-lipoxygenase genes $\mathrm{ZmLOX} 4$ and $\mathrm{ZmLOX} 5$ of maize. Planta 231, $1425-37$.

Pauletti PM, Araujo AR, Young MCM, Giesbrecht AM, Bolzani VS, 2000. nor-Lignans from the leaves of Styrax ferrugineus (Styracaceae) with antibacterial and antifungal activity. Phytochemistry 55, 597-601.

Penninck I, Thomma BP, Buchala A, Metraux JP, Broekaert WF, 1998. Concomitant activation of jasmonate and ethylene response pathways is required for induction of a plant defense. The Plant Cell 10, 210313.

Piesik D, Miler N, Lemańczyk G, Bocianowski J, Buszewski B, 2015. Botrytis cinerea infection in three cultivars of chrysanthemum in 'Alchimist' and its mutants: volatile induction of pathogen-infected plants. Scientia Horticulturae 193, 127-35.

Pinto E, Queiroz MJ, Vale-Silva LA et al., 2008. Antifungal activity of synthetic di(hetero)arylamines based on the benzo[b]thiophene moiety. Bioorganic \& Medicinal Chemistry 16, 8172-7.

Presello DA, Iglesias J, Botta G, Reid LM, Lori GA, Eyhérabide GH, 2006. Stability of maize resistance to ear rots caused by Fusarium 
graminearum and F. verticillioides in Argentinian and Canadian environments. Euphytica 147, 403-7.

Presello DA, Iglesias J, Botta G, Eyhérabide GH, 2007. Severity of Fusarium ear rot and concentration of fumonisin in grain of Argentinian maize hybrids. Crop Protection 26, 852-5.

Presello DA, Iglesias J, Botta G, Eyherabide G, 2008. Effect of disease severity on yield and grain fumonisin concentration of maize hybrids inoculated with Fusarium verticillioides. Crop Protection 27, 572-6.

Prost I, Dhondt S, Rothe G, 2005. Evaluation of the antimicrobial activities of plant oxylipins supports their involvement in defense against pathogens. Plant Physiology 139, 1902-13.

Salae AW, Karalai C, Ponglimanont C, Kanjana-Opas A, Yuenyongsawad S, 2010. Naphthalene derivatives from Diospyros wallichii. Canadian Journal of Chemistry 88, 922-7.

Scala V, Giorni P, Cirlini M, Ludovici M, 2014. LDS1-produced oxylipins are negative regulators of growth, conidiation and fumonisin synthesis in the fungal maize pathogen Fusarium verticillioides. Frontiers in Microbiology 5, 669.
Turner JG, Ellis C, Devoto A, 2002. The jasmonate signal pathway. The Plant Cell 14, 153-64.

Zeringue HJ, Brown RL, Neucere JN, Cleveland TE, 1996. Relationship between C6-C12 alkanal and alkenal volatile contents and resistance of maize genotypes to Aspergillus flavus and aflatoxin production. Journal of Agricultural and Food Chemistry 44, 403-7.

\section{Supporting Information}

Additional Supporting Information may be found in the online version of this article at the publisher's web-site.

Table S1. Gene-specific primers used for qRT-PCR analysis.

Table S2. Relative amounts and identification of volatile compounds released from grains of six Fusarium verticillioides inoculated (I) and non-inoculated (NI) maize genotypes.

Table S3. Relative amounts and identification of volatile compounds released from silks of Fusarium verticillioides inoculated (I) and noninoculated (NI) L4674 and L4637 maize genotypes. 